\title{
Assessing the Flow Field around an Oblong Bridge Pier. Vectrino Acquisition Time Sensitivity Analysis
}

\author{
Ana Margarida BENTO ${ }^{1,2, \bowtie}$, João Pedro $\mathrm{PEGO}^{2}$, Lúcia $\mathrm{COUTO}^{1}$, \\ and Teresa VISEU ${ }^{1}$ \\ ${ }^{1}$ National Laboratory of Civil Engineering, Lisboa, Portugal \\ ${ }^{2}$ Faculty of Engineering of the University of Porto, Porto, Portugal \\ $\triangle$ ana.bento@fe.up.pt
}

\begin{abstract}
Local scour has been widely identified as one of the primary threats to bridge pier stability. To better understand how the turbulent flow field modified by bridge piers interacts with the bed surface, it is relevant to assess the flow structures that are potentially sufficient to remove the bed material. Thus, the accuracy and measurement quality in estimating the flow field is of primordial importance, mainly in a well-controlled laboratory environment. In the present study, time-averaged velocities and Reynolds shear stresses were measured by using a highresolution acoustic velocimeter. Due to the highly turbulent nature of the flow, an assessment of the signal acquisition time's influence on the statistics of turbulent quantities is performed, including the inherent uncertainties regarding the flow field measurement technique. The results showed that the required sampling time for mean and fluctuation velocities, and the lateral Reynolds shear stress, differ by one order of magnitude.
\end{abstract}

Keywords: acquisition time, downlooking vectrino, statistical parameters, velocity measurements.

\section{INTRODUCTION}

The presence of a scour hole at the vicinity of a pier radically alters the turbulent flow field and its hydrodynamic characteristics. For instance, the bed shear stress and turbulence quantities are strengthened, which induces further sediment transport and scour development at the pier vicinity. The increased horseshoe vortex and the turbulent energy indicate that different mechanisms trigger the flow field structure when a scour hole is present, requiring further research to deepen the understanding of the pier scouring process (Li et al. 2020). 
The measurement quality of the turbulent flow field around a bridge pier model, using a high-resolution acoustic velocimeter (also called vectrino), is strongly influenced by three main factors: (i) the signal acquisition time, (ii) the type of seeding particles, and (iii) the alignment of the probe. The current work focuses on the assessment of the signal acquisition time for measuring the flow field and its influence on the statistics of turbulence characteristics.

\section{ACQUISITION TIME INFLUENCE}

Flow and turbulence characteristics at an eroded bed stage around a $0.14 \mathrm{~m}$ wide oblong pier at the flume mid-plane surface were assessed, with respect to the vectrino signal acquisition time, in order to check the time needed to get stable time-averaged values. The local scouring experiment belongs to a campaign composed of six fixed bed experiments performed in a recirculating tilting flume, located in the National Laboratory of Civil Engineering (LNEC), in Lisbon, Portugal (Bento 2021). An acquisition of 30 min with a 4-beam down-looking probe, with $200 \mathrm{~Hz}$ of sampling frequency (totalizing 1800 data samples), in a point situated at an upstream distance of $0.09 \mathrm{~m}$ from the front edge of the bridge pier $(0.20 \mathrm{~L}$ and $1.5 \mathrm{~W}$, being $L$ the total pier length, perpendicular to the main flow direction, and $W$ the effective pier width), and at a distance of $0.223 \mathrm{~m}$ from the bed surface, were used for this work. The experiment was performed at steady flow conditions and with the introduction of seeding particles into the flow.

Measurements were filtered using ExploreV to reject points with a correlation coefficient less than $70 \%$ and signal-to-noise ratio (SNR) less than $15 \mathrm{~dB}$ for ensuring the reliability of instantaneous velocity data (Beheshti and Ataie-Ashtiani 2010). The recorded signals were characterized by a mean correlation value of $90 \%$ and a mean SNR of $22 \mathrm{~dB}$. A sensitivity analysis of the sampling time for measuring the time-averaged streamwise velocity and fluctuation $\left(\bar{u}\right.$ and $\overline{u^{\prime}}$, respectively), and the lateral Reynolds shear stress $\left(\overline{u^{\prime} v^{\prime}}\right)$ was undertaken.

The time needed for the stabilization of $\bar{u}$ and $\bar{u}^{\prime}$ was lower than one order of magnitude for $\overline{u^{\prime} v^{\prime}}$. After 10 seconds, $\bar{u}$ indicated a relative difference of less than $2 \%$, when compared with the time-average value for 0.5 hours of the down-looking vectrino acquired signal. For $\overline{u^{\prime}}$, a relative difference of $2 \%$ was attained after 88 seconds. The variable that required longer time to stabilize was the lateral Reynolds shear stress. For this case, a relative difference of $5 \%$ was ensured after 297 seconds of acquisition. These values are in line with studies in the literature, which acquisition times range from 60 to 300 seconds (Li et al. 2020, among others).

Acknowledgments. We express our gratitude to the Portuguese Foundation for Science and Technology (FCT) for their financial support through the Ph.D. scholarship PD/BD/127798/2016 of the first author in the framework of INFRARISK Doctoral Program.

\section{References}

Beheshti, A.A., and B. Ataie-Ashtiani (2010), Experimental study of three-dimensional flow field around a complex bridge pier, J. Eng. Mech. 136, 2, 143-154, DOI: 10.1061/(ASCE)EM.19437889.0000073.

Li, J., Y. Yang, and Z. Yang (2020), Influence of scour development on turbulent flow field in front of a bridge pier, Water 12, 9, 2370, DOI: 10.3390/w12092370.

Bento, A.M. (2021), Risk-based analysis of bridge scour prediction, Ph.D. Thesis, Faculdade de Engenharia da Universidade do Porto, Porto, Portugal. 